\title{
Screening for Gonorrhea: Recommendation Statement
}

\section{U.S. Preventive Services Task Force}

Members of the U.S. Preventive Services Task Force* are Ned Calonge, MD, MPH, Chair, USPSTF (Chief Medical Officer and State Epidemiologist, Colorado Department of Public Health and Environment, Denver, Colo), Diana B. Petitti, MD, MPH, Vice-chair, USPSTF (Senior Scientific Advisor for Health Policy and Medicine, Regional Administration, Kaiser Permanente Southern California, Pasadena, Calif), Janet D. Allan, $\mathrm{PbD}, \mathrm{RN}, \mathrm{CS}$ (Dean, School of Nursing, University of Maryland, Baltimore, Baltimore, Md), Alfred O. Berg, MD, MPH (Professor and Chair, Department of Family Medicine, University of Washington, Seattle, Wasb); Paul S. Frame, MD (Family Physician, Tri-County Family Medicine, Cobocton, NY, and Clinical Professor of Family Medicine, University of Rochester, Rochester, NY), Joxel Garcia, MD, MBA (Deputy Director, Pan American Health Organization, Washington, DC) Leon Gordis, MD, MPH, DrPH (Professor, Epidemiology Department, Jobns Hopkins Bloomberg School of Public Health, Baltimore, Md); Kimberly D. Gregory, MD MPH (Director, Women's Healtb Services Researcb and Maternal-Fetal Medicine, Department of Obstetrics and Gynecology, Cedars-Sinai Medical Center, Los Angeles, Calif); Russell Harris, MD, MPH (Professor of Medicine, Sheps Center for Health Services Research, University of Nortb Carolina School of Medicine, Chapel Hill, NC), Mark S. Jobnson, MD, MPH (Professor and Chair, Department of Family Medicine, University of Medicine and Dentistry of New Jersey-New Jersey Medical School, Newark, NJ); Jonathan D. Klein, MD, MPH (Associate Professor, Department of Pediatrics, University of Rochester School of Medicine, Rochester, $N Y)_{i}$ Carol Loveland-Cherry, $\mathrm{PbD}, \mathrm{RN}$ (Executive Associate Dean, Office of Academic Affairs, University of Michigan School of Nursing, Ann Arbor, Mich), Virginia A. Moyer, MD, MPH (Professor, Department of Pediatrics, University of Texas Health Science Center, Houston, Tex), Judith K. Ockene, PbD (Professor of Medicine and Chief of Division of Preventive and Bebavioral Medicine, University of Massacbusetts Medical School, Worcester, Mass), Albert L. Siu, MD, MSPH (Professor and Chairman, Brookdale Department of Geriatrics and Adult Development, Mount Sinai Medical Center, New York, NY); Steven M. Teutsch, MD, MPH (Executive Director, Outcomes Research and Management, Merck \& Company, Inc., West Point, Pa), and Barbara P. Yawn, MD, MSc (Director of Research, Olmstead Research Center, Rochester, Minn).

* Members of the Task Force at the time tbis recommendation was finalized. For a list of current Task Force members, go to bttp://www.abrq.gov/clinic/uspstfab.btm.

\section{CORRESPONDING AUTHOR}

Ned Calonge, MD, MPH

Chair, US Preventive Services Task Force c/o Program Director, USPSTF

Agency for Healthcare Research and Quality 540 Gaither Road, Rockville, MD 20850 uspstf@ahrq.gov.
Ann Fam Med 2005;3:263-267. DOI: 10.1370/afm.337.

\section{SUMMARY OF RECOMMENDATIONS}

7 he U.S. Preventive Services Task Force (USPSTF) recommends that clinicians screen all sexually active women, including those who are pregnant, for gonorrhea infection if they are at increased risk for infection (that is, if they are young or have other individual or population risk factors; see Clinical Considerations for further discussion of risk factors). B recommendation.

Women with asymptomatic gonorrbea infection bave bigh morbidity due to pelvic inflammatory disease, ectopic pregnancy, and chronic pelvic pain. Pregnant women with gonorrbea infection are at risk for preterm rupture of membranes, preterm labor, and chorioamnionitis. There is fair evidence that screening tests can accurately detect gonorrhea infection and good evience that antibiotics can cure gonorrbea infection. There is fair evidence that screening pregnant women at bigh risk for gonorrbea, including women at bigh risk because of younger age, may prevent other complications associated with gonococcal infection during pregnancy, such as preterm delivery and chorioamnionitis. Potential barms of screening and treatment for gonorrbea include false-positive test results, anxiety, and unnecessary antibiotic use. There is insufficient evidence (due to a lack of studies) to quantify the magnitude of these potential harms. The USPSTF judges the magnitide of the potential harms to be small. The USPSTF concludes that the benefits of screening women at increased risk for gonorrbea infection outweigh the potential harms.

The USPSTF found insufficient evidence to recommend for or against routine screening for gonorrhea infection in men at increased risk for infection (see Clinical Considerations for discussion of risk factors). I recommendation.

The morbidity from undiagnosed and untreated genital gonorrbea infection is lower in men than in women. Clinical symptoms are more likely to lead to diagnosis and treatment in men, thus, the prevalence of asymptomatic infection in men is lower. There is fair evidence that noninvasive screening tests can accurately detect gonorrbea infection and good evidence that antibiotics cure gonorrbea infection. Potential barms of screening and treatment for gonorrbea include false-positive test results, anxiety, and unnecessary antibiotic use. There is insufficient evidence (due to a lack of studies) to quantify the magnitude of these potential barms. The USPSTF judges the magnitide of the potential harms of screening men for gonorrbea to be small. Given the low prevalence of asymptomatic infection in men, the USPSTF could not determine the balance of benefits and barms of screening for gonorrbea infection in men at increased risk for infection.

The USPSTF recommends against routine screening for gonorrhea infection in men and women who are at low risk for infection (see Clinical Considerations for discussion of risk factors). D recommendation.

There is a low prevalence of gonorrbea infection in the general population and conseduently a low yield from screening. Thus, the USPSTF concludes that potential harms of screening (ie, false-positive test results and labeling) in low-prevalence populations outweigh the benefits.

The USPSTF found insufficient evidence to recommend for or against routine screening for gonorrhea infection in pregnant women who are not 
at increased risk for infection (see Clinical Considerations for discussion of risk factors). I recommendation.

The prevalence of gonorrbea infection in pregnant women who are not at increased risk for infection is low. The USPSTF could not determine the balance between benefits and barms of screening for gonorrbea in pregnant women who are not at increased risk for infection.

The USPSTF strongly recommends prophylactic ocular topical medication for all newborns against gonococcal ophthalmia neonatorum. A recommendation.

There is good evidence that blindness due to gonococcal ophthalmia neonatorum bas become rare in the United States since the implementation of universal preventive medication of infants.

\section{CLINICAL CONSIDERATIONS}

- Women and men under the age of 25 yearsincluding sexually active adolescents — are at highest risk for genital gonorrhea infection. Risk factors for gonorrhea include a history of previous gonorrhea infection, other sexually transmitted infections, new or multiple sexual partners, inconsistent condom use, sex work, and drug use. Risk factors for pregnant women are the same as for nonpregnant women. Prevalence of gonorrhea infection varies widely among communities and patient populations. African Americans and men who have sex with men have a higher prevalence of infection than the general population in many communities and settings.

- Individual risk depends on the local epidemiology of disease. Local public health authorities provide guidance to clinicians to help identify populations who are at increased risk in their communities. In communities with a high prevalence of gonorrhea, broader screening of sexually active young people may be warranted, especially in settings serving individuals who are at increased risk. Additionally, clinicians may want to consider other population-based risk factors, including residence in urban communities and communities with high rates of poverty, when making screening decisions. Low community prevalence of gonorrhea infection may justify more targeted screening.

- Screening is recommended at the first prenatal visit for pregnant women who are in a high-risk group for gonorrhea infection. For pregnant patients who are at continued risk, and for those who acquire a new risk factor, a second screening should be conducted during the third trimester. The optimal interval for screening in the nonpregnant population is not known.

- Vaginal culture remains an accurate screening test when transport conditions are suitable. Newer screening tests, including nucleic acid amplification tests and nucleic acid hybridization tests, have demonstrated improved sensitivity and comparable specificity when compared with cervical culture. Some newer tests can be used with urine and vaginal swabs, which enables screening when a pelvic examination is not performed

- Appropriate treatment of gonorrhea infection and administration of prophylactic medication to newborns have been outlined by the Centers for Disease Control and Prevention (CDC) (http://www.cdc. gov/std/treatment/42002TG.htm\#Gonococcal). Genital infection in men and women may be treated with a third-generation cephalosporin or fluoroquinolone, and pregnant women may be treated with third-generation cephalosporins. Because of emerging fluoroquinolone resistance, the CDC issued new treatment guidelines in 2004 recommending that men who have sex with men and those who acquired an infection in California, Hawaii, or Asia not be treated with fluoroquinolone antibiotics. If clinicians have not concurrently screened for chlamydial infection, the CDC recommends presumptive treatment for chlamydia at the time of treatment for gonorrhea. In order to prevent recurrent transmission, partners of infected individuals should be tested and treated if infected, or treated presumptively.

- Gonorrhea is a nationally reportable condition. More complete reporting of gonorrhea cases to public health authorities will permit more accurate estimations of gonorrhea prevalence. Improved information will allow clinicians to screen for gonorrhea in ways that improve the balance between benefits and harms for their patients.

- Research priorities for gonorrhea screening include greater understanding of the benefits of screening men at increased risk, especially men who have sex with men, and the role of reporting on gonorrhea rates and testing priorities.

- See other USPSTF recommendations on screening for sexually transmitted infections (chlamydial infection, hepatitis $\mathrm{B}$ and $\mathrm{C}$ virus infection, HIV, genital herpes simplex, and syphilis) at http://www.ahrq. gov/clinic/cps3dix.htm\#infectious.

\section{DISCUSSION}

Infection because of Neisseria gonorrboeae remains the second most common reportable disease in the United States, the first being Chlamydia trachomatis. In women, gonorrhea is a major cause of cervicitis and pelvic inflammatory disease. Pelvic inflammatory disease, in turn, can lead to ectopic pregnancy, infertility, and chronic pelvic pain. Gonorrhea in pregnancy is associated with adverse outcomes, including chorioamnionitis, premature rupture of membranes, and preterm labor. Perinatal transmission to infants can cause severe conjunctivitis resulting in blindness if untreated and, rarely, sepsis with associated meningitis, endocarditis, 
or arthritis. In men, gonorrhea can result in symptomatic urethritis, epididymitis, and prostatitis. ${ }^{1}$ Emerging evidence suggests gonococcal infection facilitates susceptibility to and transmission of HIV in both men and women. ${ }^{2}$

In 2003, the rate of reported gonorrhea in the United States was 116.2 cases per a population of 100,000 . With decreasing rates each year since 1999, this marks the lowest rate of gonorrhea ever reported by the CDC. Nonetheless, only 8 states had gonorrhea rates below the Healthy People 2010 national target of 19 cases per a population of 100,000 . The prevalence of gonorrhea varies widely among regions of the country, with the South reporting the highest rates (149.8 cases per 100,000 in 2003). Rates in the South, however, have been decreasing steadily since 1999, while rates in the West have been increasing. Both rates and changes in rates differ among racial and ethnic groups.

Reported cases are rising among whites and Hispanics, and decreasing among African Americans. The rate among African Americans (655.8 per 100,000 population), however, still remains 20 times higher than for whites. As in past years, the rate of gonorrhea reported in 2003 remains highest among women aged 15 to 24 years and men aged 20 to 24 years. $^{3}$

While assessing individual risk factors provides valuable information to help determine whom to screen, clinicians should carefully consider the local epidemiology of gonorrhea infection in developing screening programs. National, state, and county-level sexually transmitted infection surveillance data are summarized by the CDC annually. Clinicians may wish to consult with their local health departments to obtain information more relevant to their specific communities and practices.

The USPSTF examined evidence published from 1996 to 2004 to determine the efficacy of gonorrhea screening in decreasing gonorrhea-related morbidity and mortality in the general population, those at increased risk, and pregnant women. In addition, the USPSTF reviewed the literature for new evidence concerning the harms of prophylactic medication to prevent gonococcal ophthalmia neonatorum. While including key questions concerning individual risk factors, this review did not include a full review of issues surrounding screening for gonorrhea infection in men who have sex with men. Issues that were not fully reviewed include choice of test for screening at rectal and pharyngeal sites and the potential role of screening for gonorrhea in reducing transmission of HIV. Although the gonorrhea prevalence outside of HIV care settings among men who have sex with men has not been widely studied, other groups have developed more specific recommendations for men who have sex with men. ${ }^{1,4}$
Screening for genital gonorrhea infection can be accomplished using culture, nucleic acid amplification tests, and nucleic acid hybridization tests (nucleic acid probes). Culture isolates can be collected from endocervical swabs in women and urethral swabs in men. Culture specimen specificity is $100 \%$ when culture isolates are speciated to differentiate Neisseria gonorrboeae from other organisms (because most studies define culture as the gold standard); however, the sensitivity of culture varies widely, ranging from $61.8 \%$ to $92.6 \%$, but remains high when transport conditions are suitable. Sensitivity for nucleic acid amplification tests ranges from $66.7 \%$ to $100 \%$, with specificity ranging from $93.9 \%$ to $100 \%$. Nucleic acid amplification tests may be used with urine specimens in addition to endocervical and urethral swabs, and single specimens can be used to test for chlamydia as well as gonorrhea. Vaginal swabs may also be used with certain nucleic acid amplification tests. Nucleic acid probes have reported sensitivity ranging from $54 \%$ to $100 \%$, with specificity ranging from $96.8 \%$ to $100 \%$. Nucleic acid probes may also be used to test for gonorrhea and chlamydia in a single specimen and may be stored for up to 7 days without refrigeration. ${ }^{5}$ Overall, newer tests have demonstrated sensitivity and specificity comparable to cervical culture, and compare better when transport conditions are not suitable for culture. Nucleic acid amplification tests can be used with urine and vaginal swabs, which enable screening when a pelvic examination is not performed. Nucleic acid amplification tests, however, have lower sensitivity when performed using a urine specimen.

Antibiotic therapy is highly effective in eliminating urogential Neisseria gonorrboeae infections. Recommended treatment for uncomplicated urogenital gonococcal infections from the CDC include one of the following antibiotic regimens: cefixime, $400 \mathrm{mg}$ orally in a single dose; ceftriaxone, $125 \mathrm{mg}$ intramuscularly in a single

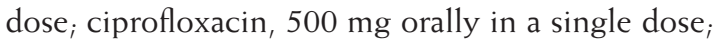
ofloxacin, $400 \mathrm{mg}$ orally in a single dose; or levofloxacin, $250 \mathrm{mg}$ orally in a single dose. ${ }^{1}$ Pregnant women should be treated with a cephalosporin-based regimen. Because of increased prevalence of resistant organisms, fluoroquinolones should not be used to treat men who have sex with men and patients whose infections were acquired in California, Hawaii, Asia, or other areas with increased resistance to fluorquinolones. ${ }^{6}$ To prevent gonococcal ophthalmia neonatorum, a prophylactic agent should be instilled into the eyes of all newborn infants; this procedure is required by law in most states. Prophylactic regimens include a single application of silver nitrate $(1 \%)$ aqueous solution, erythromycin $(0.5 \%)$ ophthalmic ointment, or tetracycline ophthalmic ointment $(1 \%),{ }^{1}$ all of which are effective.

No study has directly examined the harms of 


\section{APPENDIX A}

U.S. Preventive Services Task Force Recommendations and Ratings

The Task Force grades its recommendations according to one of 5 classifications $(A, B, C, D, I)$ reflecting the strength of evidence and magnitude of net benefit (benefits minus harms):

A. The USPSTF strongly recommends that clinicians provide [the service] to eligible patients. The USPSTF found good evidence that [the service] improves important health outcomes and concludes that benefits substantially outweigh harms.

B. The USPSTF recommends that clinicians provide [the service] to eligible patients. The USPSTF found at least fair evidence that [the service] improves important health outcomes and concludes that benefits outweigh harms.

C. The USPSTF makes no recommendation for or against routine provision of [the service]. The USPSTF found at least fair evidence that [the service] can improve health outcomes but concludes that the balance of benefits and harms is too close to justify a general recommendation.

D. The USPSTF recommends against routinely providing [the service] to asymptomatic patients. The USPSTF found at least fair evidence that [the service] is ineffective or that harms outweigh benefits.

I. The USPSTF concludes that the evidence is insufficient to recommend for or against routinely providing [the service]. Evidence that [the service] is effective is lacking, of poor quality, or conflicting, and the balance of benefits and harms cannot be determined.

screening or treatment for gonorrhea infection. Potential harms of screening may include opportunity costs to the clinician and patient (time, resources, etc) and false-positive test results that may lead to stress, labeling, and further testing. Even using a test with a specificity of $99 \%$ in a population at high risk for gonorrhea with a prevalence of $0.5 \%$, two thirds of positive screening tests would be expected to be false-positive results. Harms of treatment include adverse drugrelated effects.

In addition to research on the potential harms of screening, research is needed to provide direct evidence that screening is associated with improved health outcomes. Specifically, studies are needed to evaluate screening criteria for men, including men who have sex with men, and pregnant and nonpregnant women. Additional research is needed to determine optimal screening intervals for these populations as well as for screening after treatment. High-quality cost-effectiveness studies of current clinical options, including screening criteria and types of diagnostic tests, will also help inform future gonorrhea screening programs.

\section{RECOMMENDATIONS OF OTHER GROUPS}

The American Academy of Family Physicians (AAFP) and the American College of Obstetricians and Gynecologists (ACOG) recommend screening sexually active women, including adolescents, at high risk for gonorrhea. ${ }^{7,8}$ The AAFP, ACOG, and the American Academy of Pediatrics (AAP) recommend screening pregnant women at risk for gonorrhea., ${ }^{79}$ The Depart-

\section{APPENDIX B}

U.S. Preventive Services Task Force Strength of Overall Evidence

The USPSTF grades the quality of the overall evidence for a service on a 3-point scale (good, fair, poor):

Good

Evidence includes consistent results from well-designed, well-conducted studies in representative populations that directly assess effects on health outcomes.

Fair

Evidence is sufficient to determine effects on health outcomes, but the strength of the evidence is limited by the number, quality, or consistency of the individual studies, generalizability to routine practice, or indirect nature of the evidence on health outcomes.

Poor

Evidence is insufficient to assess the effects on health outcomes because of limited number or power of studies, important flaws in their design or conduct, gaps in the chain of evidence, or lack of information on important health outcomes.

ment of Defense recommends screening for gonorrhea in all pregnant women based on a presumption of potential risk within their system. ${ }^{10}$ The AAFP and AAP recommend routine prophylaxis for newborns against gonococcal ophthalmia neonatorum. ${ }^{7,11}$ The Centers for Disease Control and Prevention (CDC) has published recommendations on diagnostic testing for gonorrhea that support the use of culture as a test for use when screening. ${ }^{12}$ The CDC also recommends that clinicians consider all positive screening tests presumptive evidence of infection and consider additional testing when screening in low-prevalence populations. ${ }^{12}$ In its 2002 Clinical Treatment Guidelines, the CDC recommends that all sexually active men who have sex with men be screened at least annually for genital gonorrhea and also for pharyngeal and rectal infection if at risk due to exposure. ${ }^{1}$ The Infectious Disease Society of America recommends that all HIV-positive individuals be screened for gonorrhea. ${ }^{4}$

This statement summarizes the U.S. Preventive Services Task Force (USPSTF) recommendation on screening for gonorrhea and the supporting scientific evidence, and updates the 1996 recommendations contained in the Guide to Clinical Preventive Services, second edition. ${ }^{13}$ Explanations of the ratings and of the strength of overall evidence are given in Appendix A and Appendix $\mathrm{B}$, respectively. The complete information on which this statement is based, including evidence tables and references, is included in the evidence synthesis ${ }^{5}$ on this topic, available through the USPSTF Web site (http:// www.preventiveservices.ahrq.gov). The recommendation is also posted on the Web site of the National Guideline Clearinghouse ${ }^{\mathrm{TM}}$ (http://www.guideline.gov).

Recommendations made by the USPSTF are independent of the U.S. Government. They should not be construed as an official position of AHRQ or the U.S. Department of Health and Human Services. 
To read or post commentaries in response to this article, see it online at http://www.annfammed.org/cgi/content/full/3/3/263.

Key words: Gonorrhea; mass screening; practice guidelines

\section{References}

1. Sexually transmitted diseases treatment guidelines 2002. Centers for Disease Control and Prevention. MMWR Recomm Rep. 2002;5 (RR-6): 1-78.

2. Farley TA, Cohen DA, Wu SY, Besch CL. The value of screening for sexually transmitted diseases in an HIV clinic. J Acquir Immune Defic Syndr. 2003;33:642-648.

3. STD Surveillance 2003. Centers for Disease Control and Prevention. Available at: http://www.cdc.gov/std/stats/gonorrhea.htm. Accessed 17 December 2004.

4. Aberg JA, Gallant JE, Anderson J, et al; HIV Medicine Association of the Infectious Diseases Society of America. Primary care guidelines for the management of persons infected with human immunodeficiency virus: recommendations of the HIV Medicine Association of the Infectious Diseases Society of America. Clin Infect Dis. 2004;39:609-629.

5. Glass N, Nelson H, Villemyer K. Screening for Gonorrhea. Evidence Synthesis No. 40. (Prepared by the Oregon Evidence-based Practice Center under Contract No. 290-02-0024). Rockville, Md: Agency for Healthcare Research and Quality. May 2005. Available at: http:// www.ahrq.gov/clinic/serfiles.htm.
6. Centers for Disease Control and Prevention. Increases in fluoroquinolone-resistant neisseria gonorrhoeae among men who have sex with men --- United States, 2003, and revised recommendations for gonorrhea treatment, 2004. MMWR. 2004;53:335-338.

7. American Academy of Family Physicians. Recommendations for periodic health examinations. August 2004. Available at: http://www. aafp.org/x24975.xml. Accessed 7 March 2004

8. American College of Obstetricians and Gynecologists. ACOG Committee Opinion. Primary and preventive care: periodic assessments. Obstet Gynecol. 2003;102:1117-1124.

9. American Academy of Pediatrics, American College of Obstetricians and Gynecologists. Guidelines for Perinatal Care. 5th ed. Washington, DC: ACOG; 2002.

10. DoD/VA clinical practice guideline for management of uncomplicated pregnancy. Clinical Practice Guidelines. Available at: http://www. oqp.med.va.gov/cpg/UCP/UP_base.htm. Accessed 7 March 2005.

11. American Academy of Pediatrics. 2003 Red Book Report on the Com mittee of Infectious Diseases. 26th ed. Elk Grove Village, IL: American Academy of Pediatrics; 2003.

12. Johnson RE, Newhall WJ, Papp JR, et al. Screening tests to detect Chlamydia trachomatis and Neisseria gonorrhoeae infections-2002. MMWR Recomm Rep. 2002;51(RR-15):1-38; quiz CE1-4

13. U.S. Preventive Services Task Force. Guide to Clinical Preventive Services. 2nd ed. Washington, DC: Office of Disease Prevention and Health Promotion; 1996. 